\title{
EFEITO DE POTENCIAL DE ANODIZAÇÃO NA PROPRIEDADE FOTOELETROQUÍMICA DE NANOTUBOS $\mathrm{DE} \mathrm{TIO}_{2}^{*}$
}

Thais Cristina Lemes dos Santos ${ }^{1}$ Sherdil Khan ${ }^{2}$

Célia de Fraga Malfatti ${ }^{3}$

\begin{abstract}
Resumo
Neste trabalho será apresentado estudo sobre nanotubos de dióxido de titânio $\left(\mathrm{TiO}_{2}\right)$ sintetizados pela técnica de anodização com variação do potencial entre $10 \mathrm{~V}$ e $50 \mathrm{~V}$ durante tempo fixo de $1 \mathrm{~h}$. Além disso, para obter a estrutura cristalina deseja as amostras passaram por um tratamento térmico que foi realizado a $400{ }^{\circ} \mathrm{C}$ por $3 \mathrm{~h}$ a uma taxa de aquecimento de $10^{\circ} \mathrm{C} / \mathrm{min}$. As amostras foram caracterizadas pelas técnicas de microscopia eletrônica de varredura com emissão de campo (MEVFEG), difração de raios X (DRX), espectrofotometria no ultravioleta visível (UV-VÍS) e de espectroscopia de impedância eletroquímica (EIE). Observou-se que o potencial de anodização exerce influência obre a morfologia dos nanotubos e sobre a absorção de luz e, consequentemente, sobre o desempenho fotoeletroquímico das amostras. O melhor desempenho foi obtido a partir da amostra anodizada a $30 \mathrm{~V}$, este resultado está relacionado com a sua ótima absorção de luz e a sua baixa resistência ao transporte de carga interfacial obtido por EIE.
\end{abstract}

Palavras-chave: Dióxido de Titânio; Fotoeletrólise; Anodização; Potencial de anodização.

\section{EFFECT OF ANODIZATION POTENTIAL ON PHOTOELECTROCHEMICAL PROPERTIES OF $\mathrm{TiO}_{2}$ NANOTUBES}

\begin{abstract}
In this work the nanotubes of titanium dioxide $\left(\mathrm{TiO}_{2}\right)$ were synthesized by sonoelectrochemical anodization technique with changing anodization potential from 10 to $50 \mathrm{~V}$ for a fixed time of $1 \mathrm{~h}$. To obtain the crystalline structure the heat treatment was performed at $400^{\circ} \mathrm{C}$ for $3 \mathrm{hrs}$ for a heating rate of $10^{\circ} \mathrm{C} / \mathrm{min}$. The samples were characterized by field emission scanning electron microscopy (FE-SEM), x-ray diffraction (XRD), UV-Vis spectrophotometery and electrochemical impedance spectroscopy (EIS). It was observed that the anodizing potential influences the morphology of the nanotubes and the light absorption and therefore the photoelectrochemical performance of the prepared samples. The best performance was obtained from the sample anodized at $30 \mathrm{~V}$ that was related to its optimal light absorption and lowest interfacial charge transportation resistance obtained by EIS.
\end{abstract}

Keywords: Titanium dioxide; Photoelectrolysis; Anodization; Anodization potential.

1 Engenheira em Energia - UERGS, Mestranda em Engenharia de Materiais (PPGE3M) Universidade Federal do Rio Grande do Sul (UFRGS), Porto Alegre, RS, Brasil.

2 Bacharel em Física/Doutorado em Física, pós-doutorando em Engenharia (PPGE3M) Universidade Federal do Rio Grande do Sul (UFRGS), Porto Alegre, RS, Brasil.

3 Engenheira Metalúrgica, Doutora, Professora Dra., Departamento de Metalurgia, Universidade Federal do Rio Grande do Sul, Porto Alegre, Rio Grande do Sul, Brasil. 


\section{INTRODUÇÃO}

O atual modelo econômico, o aumento da demanda energética e o crescimento da consciência ambiental se mostram um excelente cenário para novas pesquisas em que não seja necessário o consumo de combustíveis fósseis. Nesse contexto, o estudo e a pesquisa da fotoeletrocatálise para produção de hidrogênio através da quebra molécula da água se tornam cada vez mais uma alternativa válida e atrativa, pois se trata de uma energia limpa e renovável [1].

Segundo Honda e Fujishima ao irradiar uma fonte de luz em um eletrodo de semicondutor conectado separado de um outro eletrodo dentro de água, se tornava possível a decomposição da água em oxigênio e em hidrogênio, desde que a energia da fonte de luz seja maior que a energia de Bandgap do material, e assim o comportamento fotocatalítico foi descoberto [2]. Ao irradiar o sistema, ocorre o aumento de corrente que indica a presença de fotoatividade nos eletrodos [3].

$\mathrm{Na}$ fotoeletrocatálise o fator mais importante é o semicondutor, que apresenta como função a absorbância da luz, a separação das cargas movidas pela luz e o transporte desta carga para o processo de oxidação e redução da água.

$\mathrm{O}$ oxido de titânio $\left(\mathrm{TiO}_{2}\right)$ é um material muito utilizado atualmente, devido à sua baixa densidade, alta resistência à ruptura e à corrosão, podendo ter sua utilização associada a produção de hidrogênio. No entanto, a absorbância do $\mathrm{TiO}_{2}$ é apenas na luz ultra violeta sendo essa apenas $4 \%$ da luz solar. $\mathrm{O} \mathrm{TiO}_{2}$ apresenta polimorfismo, normalmente, possui três formas cristalinas: anatase, bruquita e rutilo. [4].

Muitas nanoestruturas já foram utilizadas para fotoeletrólise, entre elas nanotubos de $\mathrm{TiO}_{2}$ se mostraram muito promissores. A característica unidimensional dos nanotubos ajuda muito no transporte de elétrons, pois devido ao contato elétrico eles apresentam uma resistividade muito menor. Nanotubos de $\mathrm{TiO}_{2}$ são obtidos, normalmente, pela técnica de anodização, pois através dela, é possível controlar a formação, o tamanho e o crescimento do óxido na superfície do substrato e por se tratar de uma técnica simples e viável [3].

Absorbância de um filme semicondutor depende muito da sua espessura, para escolher a melhor amostra de $\mathrm{TiO}_{2}$ para fotoeletrocatálise o semicondutor deve apresentar comprimento suficiente que permita absorver mais luz e ao mesmo tempo não pode ser muito espesso, caso contrário, aumentará resistência em série. Devido a isso é muito importante obter uma amostra de nanotubo de $\mathrm{TiO}_{2}$ que apresente um comprimento que permita absorver mais luz, ótima espessura para uma melhor área superficial e uma menor resistividade. Segundo Fernandes a região espectral de absorção do $\mathrm{TiO}_{2}$ não depende do comprimento dos tubos, estudos mostram que as maiores densidades de corrente são encontradas em comprimentos dos nanotubos intermediários [1].

Neste trabalho o tempo de anodização permaneceu fixo e houve a variação do potencial de anodização com o objetivo de analisar o efeito do potencial de anodização na resposta fotoeletroquímica de nanotubos de $\mathrm{TiO}_{2}$ e escolher a melhor condição para utilizar $\mathrm{TiO}_{2}$ na retroestrutura.

\section{MATERIAIS E MÉTODOS}

Para a anodização foram utilizadas amostras Titânio de $4 \mathrm{~mm}$ de espessura, $30 \mathrm{~mm}$ de diâmetro com pureza 98,6 \%, acetona (Synth, 99,5\%), NH4F (fluoreto de amônia, 
marca Synth, 98\%), EG (etileno glicol, marca Synth, 99,5\%). Para fotoeletrólise foram utilizados $\mathrm{Na}_{2} \mathrm{So}_{3}$ (sulfito de sódio) e $\mathrm{Na}_{2} \mathrm{~S}$ (sulfeto de sódio).

As amostras de Ti foram lixadas, polidas, lavadas em banho de ultrassom com água deionizada e acetona por $1 \mathrm{~h}$, só água deionizada por $1 \mathrm{~h}$, acetona por $30 \mathrm{~min}$, só água deionizada por 30 min e depois de secas foram anodizadas. A amostra de $\mathrm{Ti}$ (ânodo) e um disco de cobre (cátodo) foram colocados dentro do reator, o processo de anodização foi realizado imerso em banho de ultrassom. Como eletrólito foi utilizado uma solução aquosa de $\mathrm{EG}+10 \% \mathrm{H}_{2} \mathrm{O}+0,5 \% \mathrm{NH}_{4} \mathrm{~F}(\mathrm{~mm})$ em volume de $200 \mathrm{ml}$, a temperatura tanto do ultrassom quanto do eletrólito foi de $27^{\circ} \mathrm{C}$.

processo de anodização de cada amostra foi feito por $1 \mathrm{~h}$, nas tensões de $10 \mathrm{~V}, 20$ $\mathrm{V}, 30 \mathrm{~V}, 40 \mathrm{~V}$ e $50 \mathrm{~V}$ com uma rampa de $10 \mathrm{~V} / \mathrm{s}$ para todas as amostras. Após a anodização, as amostras passaram por tratamento térmico de $400{ }^{\circ} \mathrm{C}$ por $3 \mathrm{~h}$ em uma taxa de aquecimento de $10^{\circ} \mathrm{C} / \mathrm{min}$.

Neste trabalho as amostras de nanotubos (Nt) de $\mathrm{TiO}_{2}$ foram nomeadas da seguinte forma: $\mathrm{Nt}_{x}$, sendo $\mathrm{x}$ o potencial de anodização, por exemplo, $\mathrm{Nt}_{10}$ para amostra anodizada a $10 \mathrm{~V}$.

\subsection{Caracterização}

As imagens de Microscopia Eletrônica de Varredura com emissão de campo (MEVFEG) foram obtidas com um equipamento da marca microscopia Zeiss Auriga $\mathrm{O}$ objetivo da técnica foi de analisar o tamanho do diâmetro interno e externo, espessura da parede tanto do topo quanto da base dos nanotubos sintetizados e o seu comprimento. Foi feito um risco na amostra para verificar e medir o comprimento do nanotubo e nas áreas em que o nanotubo foi retirado, pode-se medir a espessura da parede e os diâmetros internos e externos dos nanotubos na base.

Difração de raios-X (DRX): as medidas de DRX foram realizadas em um difratômetro da marca Siemens, modelo D500, com geometria Bragg-Brentano utilizando um tubo de radiação $\mathrm{Ka}(\lambda=1.54 \AA)$, com passo de $0,05^{\circ}$, em intervalos de $5 \mathrm{~s}$ e com a região angular $2 \theta=20^{\circ}-70^{\circ}$.

Espectroscopia de UV-Visível (UV/VIS): medidas de UV/VÍS foram realizadas em um espectrofotômetro (Carry 5000) com o objetivo de caracterizar as bandas de absorção das amostras em função da tensão aplicada durante a anodização.

Medidas de fotoeletrólise: com a finalidade de avaliar o comportamento fotoeletroquímico (sob iluminação e sem iluminação a cada 5 segundos) e medir a densidade de fotocorrente gerada, foi realizada uma voltametria linear na região de potencial entre $(-1 \mathrm{~V}$ até $0,8 \mathrm{~V}$ versus $\mathrm{Ag} / \mathrm{AgCl})$. A célula fotoeletroquímica (reator) é formada por três eletrodos diferentes, como eletrodo de referência (RE) foi utilizado um eletrodo de $\mathrm{Ag} / \mathrm{AgCl}$, como contra-eletrodo (CE) foi utilizado um fio de platina, e como eletrodo de trabalho (WE) foram utilizadas as amostras de $\mathrm{Nt}_{-} \mathrm{TiO}_{2}$. O eletrólito utilizado foi uma solução aquosa de 0,35 $\mathrm{M}$ de $\mathrm{Na}_{2} \mathrm{SO}_{3}$ e 0,24 $\mathrm{M}$ de $\mathrm{Na}_{2} \mathrm{~S}$ em volume total de $200 \mathrm{ml}$. Foi utilizado um potenciostato (marca AUTOLAB, modelo PGSTAT $100 \mathrm{~N})$ para realização das medidas de voltametria linear, com uma velocidade de varredura de $10 \mathrm{mV} / \mathrm{s}$, e intervalo $5 \mathrm{~s}$ com e sem luz. Foi utilizado um simulador de luz solar, (marca Newport, modelo 69911 e 67005) com uma lâmpada de xenônio (Xe da marca Oriel) em uma potência fixada durante a realização da medida e também foi utilizado um filtro (AM 1.5) em $100 \mathrm{~mW} . \mathrm{cm}^{-2}$ (1 sol).

Medidas espectroscopia impedância eletroquímica (EIE): as medidas de impedância eletroquímica também foram realizadas na mesma configuração de três eletrodos com 1 sol de iluminação no potencial de circuito aberto. As medidas de impedância 
foram realizadas sob iluminação utilizando um potenciostato (marca AUTOLAB, modelo PGSTAT 100N) com amplitude de $10 \mathrm{mV}$ e frequência no intervalo de 100 $\mathrm{kHz}$ a $100 \mathrm{mHz}$.

\section{RESULTADOS E DISCUSSÃO}

Através das imagens obtidas pela técnica de MEV-FEG, foi possível observar as medidas referentes ao diâmetro interno e externo (figuras 1 e 2) e a espessura da parece ambos no topo (figura 1) e na base (figura 2), e o comprimento (figura 3) dos nanonutos sintetizados.
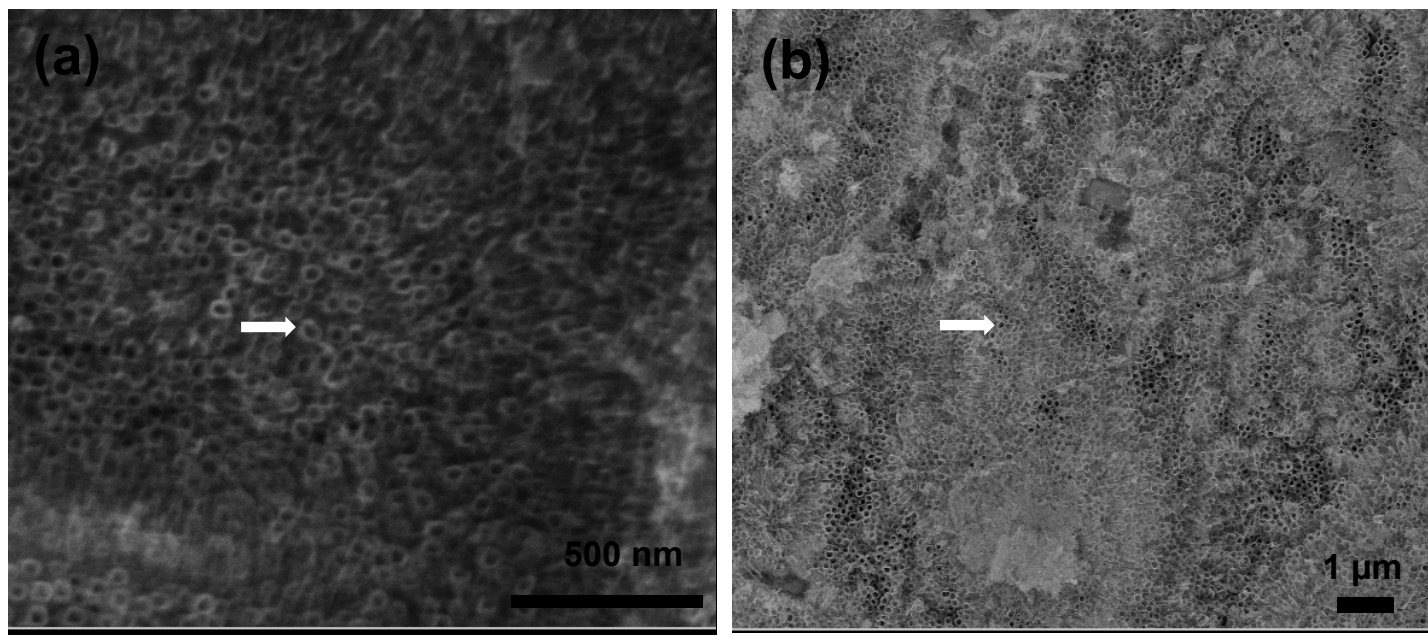

Figura 1. Imagens do topo dos nanotubos das amostras a) $\mathrm{Nt}_{10}$ b) $\mathrm{Nt}_{50}$

As imagens da figura 1 mostram a superfície do topo das amostras com a variação do potencial, podemos analisar que há um crescimento diretamente proporcional relacionado com o potencial aplicado. Esse crescimento é observado tanto na espessura da parede do topo do nanotubo, quanto nas medidas dos diâmetros interno e externo também do topo do nanotubos.
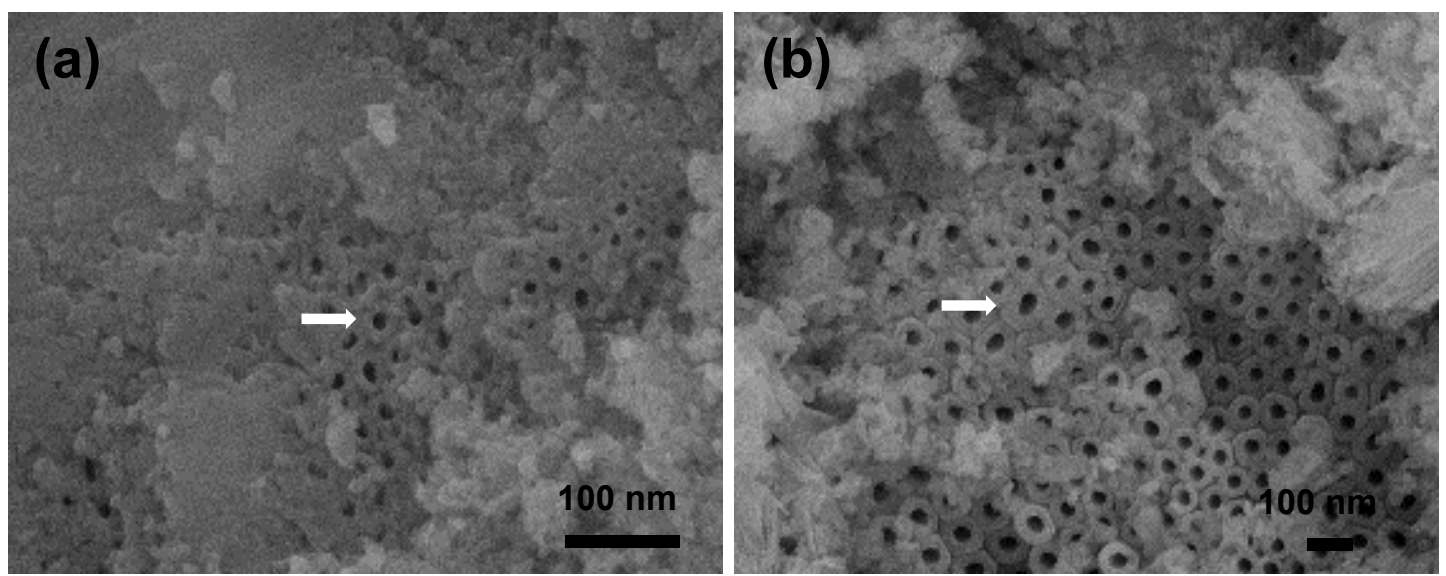

Figura 2. Imagens da base dos nanotubos das amostras a) $\mathrm{Nt}_{10}$ b) $\mathrm{Nt}_{50}$

As imagens da figura 2 mostram a base dos nanotubos sintetizados a partir da variação do potencial aplicado, podemos observar a espessura do diâmetro interno e externo e da parede das amostras. Essas imagens foram feitas nas regiões onde amostra foi riscada. As medidas da base também mostram o crescimento diretamente proporcional ao potencial aplicado. 
A tabela 1 relaciona os valores encontrados para a espessura da parede, diâmetro interno e diâmetro externo, essas medidas foram obtidas tanto da base quanto do topo dos nanotubos e também foi mensurada a medida do comprimento dos nanotubos sintetizados.

Tabela 1. Valores mensurados das medidas dos nanotubos sintetizados

\begin{tabular}{lcccc}
\hline Amostras & $\begin{array}{c}\text { Espessura da } \\
\text { parede topo } \\
(\mathbf{n m})\end{array}$ & $\begin{array}{c}\text { Diâmetro } \\
\text { interno topo } \\
(\mathbf{n m})\end{array}$ & $\begin{array}{c}\text { Diâmetro } \\
\text { externo topo } \\
(\mathbf{n m})\end{array}$ & $\begin{array}{c}\text { Comprimento } \\
(\boldsymbol{\mu m})\end{array}$ \\
\hline $\mathrm{Nt}_{10}$ & 9,4 & 30,7 & 47,8 & 0,8 \\
\hline $\mathrm{Nt}_{20}$ & 15,1 & 55,0 & 84,0 & 1,2 \\
\hline $\mathrm{Nt}_{30}$ & 16,9 & 82,2 & 115,1 & 2,3 \\
\hline $\mathrm{Nt} 40$ & 17,5 & 117,4 & 150,6 & 3,5 \\
\hline $\mathrm{Nt} t_{50}$ & 19,0 & 128,0 & 161,2 & 5,2 \\
\hline $\mathbf{A m o s t r a s}$ & $\begin{array}{c}\text { Espessura da } \\
\text { parede base } \\
\text { (nm) }\end{array}$ & $\begin{array}{c}\text { Diâmetro } \\
\text { interno base } \\
\text { (nm) }\end{array}$ & $\begin{array}{c}\text { Diâmetro } \\
\text { externo base } \\
\text { (nm) }\end{array}$ & \\
\hline $\mathrm{Nt}_{10}$ & 29,1 & 40,3 & 101,1 & - \\
\hline $\mathrm{Nt}_{20}$ & 31,2 & 53,1 & 102,2 & - \\
\hline $\mathrm{Nt}_{30}$ & 42,2 & 61,3 & 145,9 & - \\
\hline $\mathrm{Nt}_{40}$ & 56,4 & 65,5 & 161,9 & - \\
\hline $\mathrm{Nt}_{50}$ & 61,5 & 103,9 & 225,4 & - \\
\hline
\end{tabular}

A partir dos valores obtidos e apresentados na tabela 1, podemos observar que tanto os valores da base quanto do topo apresentaram uma evolução diretamente proporcional ao potencial aplicado nos nanotubos sintetizados. A parede da base e o diâmetro externo da base se apresentam maiores, ou seja, mais grossos quando comparados aos valores medidos no topo dos nanotubos.

O comprimento dos nanotubos também apresentou relação direta com o potencial, quanto maior o potencial aplicado maior foi o comprimento do nanotubo, sendo a amostra $\mathrm{Nt}_{10}$ o nanotubo menor comprimento e $\mathrm{Nt}_{50} \mathrm{o}$ nanotubo de maior comprimento. A figura 3 mostra os nanotubos da amostra $\mathrm{Nt}_{50}$ e $\mathrm{Nt}_{20}$.
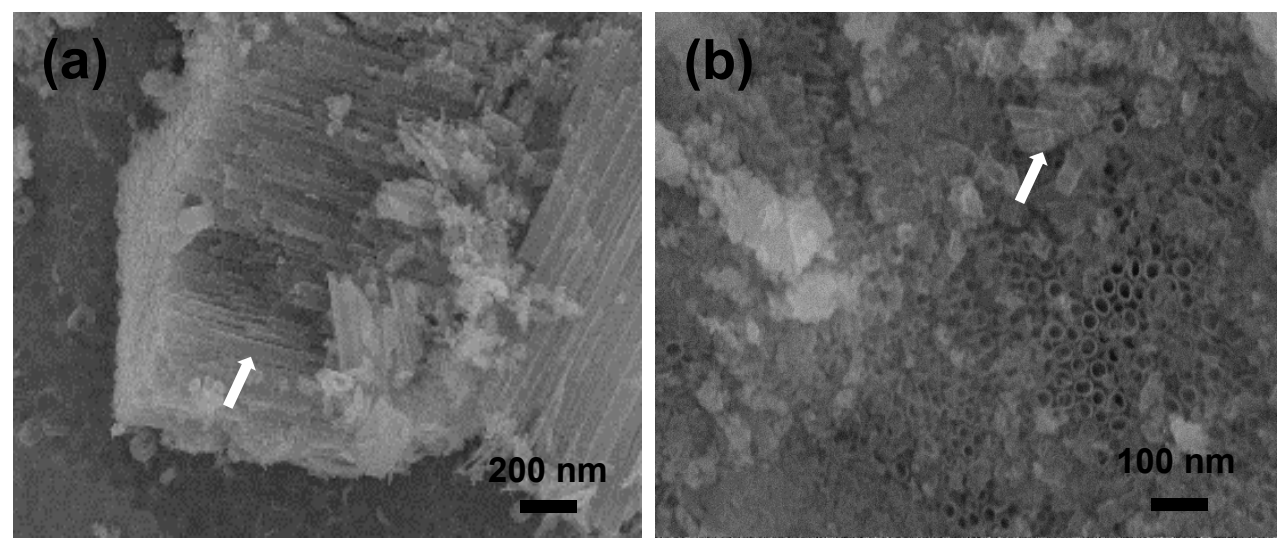

Figura 3. Imagens do comprimento dos nanotubos das amostras a) $\mathrm{Nt}_{50}$ b) $\mathrm{Nt}_{20}$

A figura 4 a) mostra difratograma de $\mathrm{DRX}$ das amostras de $\mathrm{TiO}_{2}$ após tratamento térmico a $400{ }^{\circ} \mathrm{C}$ durante $3 \mathrm{~h}$. A partir dos picos obtidos no gráfico a) foi estimado valores de intensidade máxima dos picos de $\mathrm{Ti}$ e $\mathrm{TiO}_{2}$ (anatase), conforme apresentado na tabela 2. 
Tabela 2. Valores estimados de intensidade de $\mathrm{TiO}_{2}\left(2 \theta=25,3^{\circ}\right)$ na fase anatase e $\mathrm{Ti}\left(2 \theta=40,2^{\circ}\right)$ e da intensidade relativa das amostras

\begin{tabular}{lccc}
\hline Amostras & $\mathrm{TiO}_{2}$ (anatase) & $\mathbf{~ i i}$ & $\mathbf{T i O}_{2} / \mathrm{Ti}$ \\
\hline $\mathrm{Nt}_{10}$ & 250 & 3560 & 0,070 \\
\hline $\mathrm{Nt}_{20}$ & 740 & 3375 & 0,22 \\
\hline $\mathrm{Nt}_{30}$ & 1450 & 3250 & 0,45 \\
\hline $\mathrm{Nt}_{40}$ & 2200 & 2750 & 0,8 \\
\hline $\mathrm{Nt}_{50}$ & 2625 & 2200 & 1,20 \\
\hline
\end{tabular}

Ao analisar o gráfico 4 a) e os valores estimados percebemos que conforme os picos da fase anatase $\mathrm{TiO}_{2}$ aumentam, os picos de Ti diminuem, ou seja, está ocorrendo à formação de $\mathrm{TiO}_{2}$ mais espesso de uma forma diretamente proporcional ao potencial aplicado nas amostras na anodização. Resultado compatível com o que observamos no MEV/FEG (figura 3), em que o comprimento do nanotubo aumenta conforme o potencial aplicado.
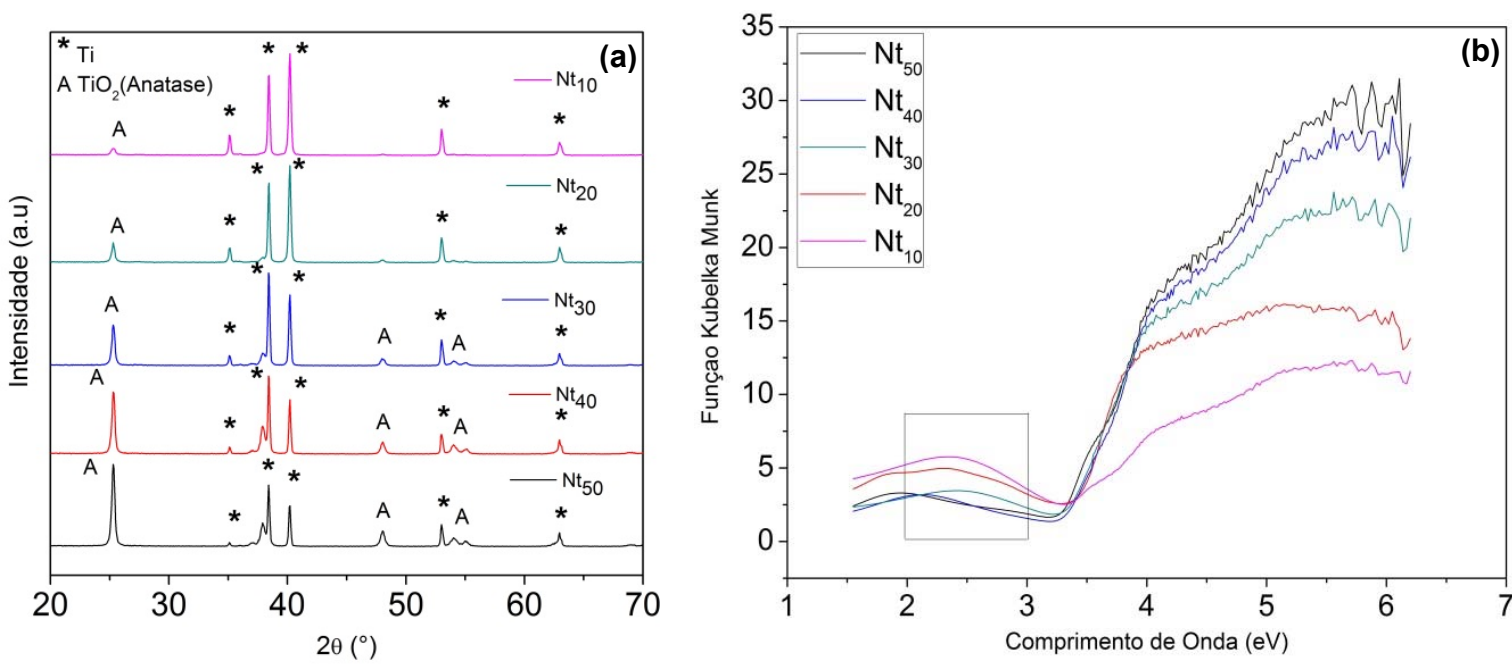

Figura 4. a) Difratogramas DRX das amostras em diferentes tensões aplicadas. b) Espectros de absorção das amostras $\mathrm{Nt}_{x}-\mathrm{TiO}_{2}$.

A figura 4 b) apresenta os resultados de UV-VíS das amostras. Pode-se observar que a amostra $\mathrm{Nt}_{50}$ apresentou absorbância máxima com valor maior em comparação as demais amostras testadas. Além disso, a amostra $\mathrm{Nt}_{10}$, apresentou o menor valor de absorção. Na região de menor energia, entre $2 \mathrm{eV}$ e $3 \mathrm{eV}$, os nanotubos de menor comprimento, apresentam flutuações que pode estar relacionado com a interferência ótica ou devido aos estados de armadilhas dentro bandgap do $\mathrm{TiO}_{2}$ [5]. Em nanotubos de maior comprimento a interferência é menor. $\mathrm{O} \mathrm{TiO}_{2}$ necessita de uma energia de bandgap de cerca de 3,2 eV para ser ativado, que corresponde à radiação UV de comprimento de onda menor do que $387 \mathrm{~nm}$ [6]. A figura 5 a) apresenta as medidas fotoeletroquímicas das amostras de $\mathrm{Nt}_{x}$ que foram obtidas aplicando diferentes tensões, é possível observar que a resposta fotoeletroquímica apresenta uma dependência da tensão empregada no processo de anodização. Para avaliar a velocidade de resposta fotoeletroquímica das amostras de $\mathrm{Nt}_{\mathrm{x}}$, a voltametria linear foi realizada com incidência de luz e com a luz bloqueada a cada cinco segundos. O rápido aumento de densidade da corrente ao irradiar a luz mostra que nanotubos indica a reposta fotoeletroquímica sob luz incidente. 

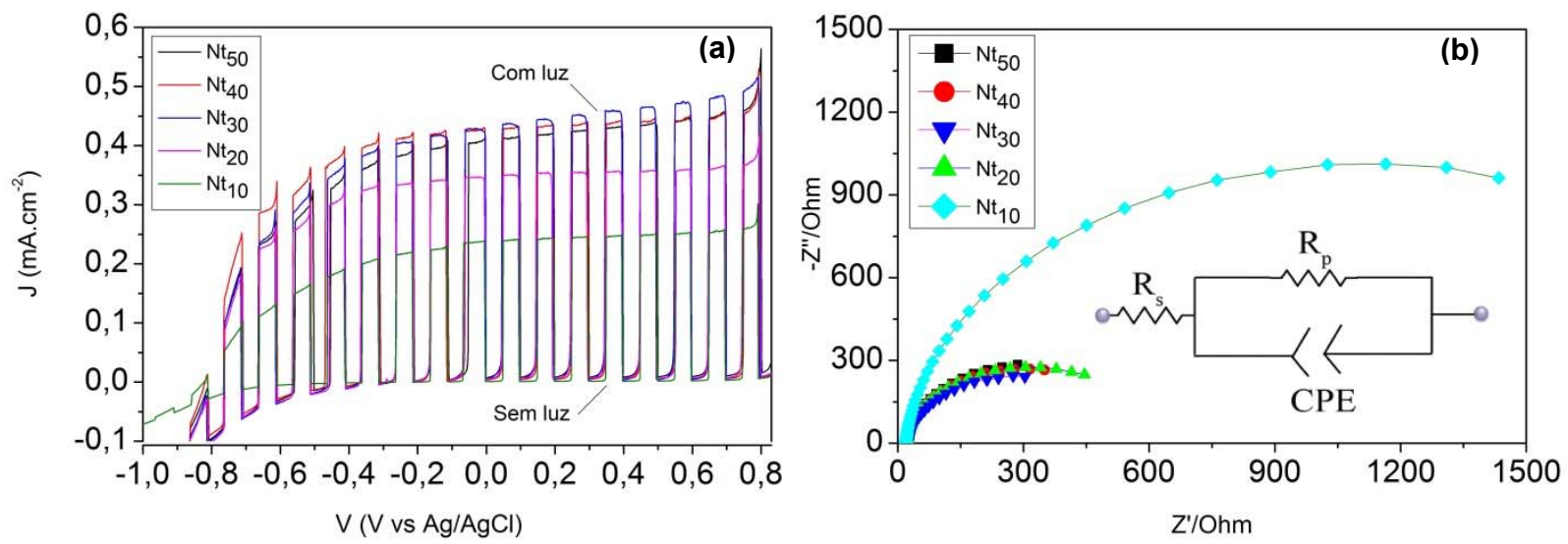

Figura 5. a) Voltametria linear das amostras de $\mathrm{Nt}_{\mathrm{x}}$, sob irradiação simulando a luz solar e com bloqueio da luz, com intervalo de cinco segundos. b) Medidas de impedância eletroquímica das amostras de $\mathrm{Nt}_{\mathrm{x}}$.

As maiores densidade de corrente (figura 5 a) foram obtidas nas amostras $\mathrm{Nt}_{30}, \mathrm{Nt}_{40}$ e Nt50. E como foi possível observar no MEV/FEG (figura 3), entre essas, a amostra que apresentou menor comprimento de nanatubo foi $\mathrm{Nt}_{30}$, por outro lado, a menor densidade de corrente foi obtida na amostra $\mathrm{Nt}_{10}$. Quando o sistema é irradiado, ocorre um aumento de corrente indicando a presença de fotoatividade nos eletrodos [7].

Para um melhor fotoeletrodo, o comprimento do nanotubo deve ter tamanho suficiente para absorver luz e deve apresentar um valor mínimo para resistividade no caminho dos elétrons até a platina [8].

Para analisar a resistividade interfacial dos nanotubos foram realizadas medidas de impedância eletroquímica. Todos os eletrodos apresentam um semicírculo que não é completo, isso mostra que a reação química que está ocorrendo na superfície dos nanotubos não é uniforme [1]. Outro fator importante é o fato dos nanotubos apresentarem morfologia porosa, conforme apresentado no MEV/FEG. Baseado nessas informações os dados de eletroquímica e impedância foram fitados em um circuito mostrado na figura $5 \mathrm{~b}$ ) que apresenta um resistor em série ( $\left.R_{s}\right)$ referente a resistência do eletrólito, um resistor paralelo $\left(R_{p}\right)$ referente à resistência da interface $\mathrm{TiO}_{2}$ /eletrólito e um elemento chamado elemento de fase constante (CPE).

A tabela 3 apresenta os valores encontrados para $R_{s}$ e $R_{p}$. Os valores de $R_{s}$ se mostram muito próximos, apresentando uma pequena variação de $19 \Omega$ a $24 \Omega$. $O$ maior valor de $\mathrm{R}_{\mathrm{p}}$ foi observado na amostra $\mathrm{Nt}_{10}$, ou seja, a interface $\mathrm{TiO}_{2}$ /eletrólico da amostra $\mathrm{Nt}_{10}$ se mostrou a mais resistiva e a amostra menos resistiva foi $\mathrm{Nt}_{30}$.

Tabela 3. Valores de Resistência $(\Omega)$ obtido pela fitting de circuito equivalente e de bandgap (eV) das amostras

\begin{tabular}{lrrc}
\hline Amostras & $\mathbf{R}_{\mathbf{s}}(\boldsymbol{\Omega})$ & $\mathbf{R}_{\mathbf{p}}(\boldsymbol{\Omega})$ & Bandgap $(\mathbf{e V})$ \\
\hline $\mathrm{Nt}_{10}$ & 19 & 2.180 & 3,32 \\
\hline $\mathrm{Nt}_{20}$ & 22 & 591 & 3,12 \\
\hline $\mathrm{Nt}_{30}$ & 24 & 522 & 3,25 \\
\hline $\mathrm{Nt}_{40}$ & 21 & 566 & 3,27 \\
\hline $\mathrm{Nt} 50$ & 22 & 604 & 3,20 \\
\hline
\end{tabular}


Na tabela 3 também podemos analisar os valores de Bandgap obtidos das amostras analisadas. A amostra $\mathrm{Nt}_{20}$ apresentou menor valor de energia de Bandgap e a amostra $\mathrm{Nt}_{10}$ apresentou maior energia.

Como observado na figura 5 a) a fotocorrente das amostras $\mathrm{Nt}_{30}, \mathrm{Nt}_{40}$ e $\mathrm{Nt}_{50}$ apresentam valores muito próximos, no entanto, a fotocorrente de $\mathrm{Nt}_{30}$ é um pouco maior do que as demais. Podemos analisar pela UV-Vís (figura 4 b) que a absorbância máxima de $\mathrm{Nt}_{40}$ e $\mathrm{Nt}_{50}$ é quase a mesma, isso significa que a saturação da absorbância para amostras obtidas com tensão de anonização acima de $30 \mathrm{~V}$.

Nanotubos com maior comprimento também apresentam maior resistência, isso significa que a partir do potencial de $30 \mathrm{~V}$, com o aumento do comprimento dos nanotubos (figura 3) a fotocorrente satura. Esses resultados mostram que a melhor corrente e o comprimento do nanotubo são fatores importantes tanto para absorver luz, quanto para apresentar alta fotocorrente.

Através dos resultados de fotocorrente e impedância eletroquímica, podemos afirmar que amostra anodizada com $30 \mathrm{~V}$ apresenta propriedades óticas e fotoeletroquímicas como um fotoeletrodo muito promissor que pode ser utilizada como um template para fazer heteroestrutura baseados em nanotubos de $\mathrm{TiO}_{2}$.

\section{CONCLUSÃO}

Foi possível verificar que o potencial de anodização tem influência direta sobre a dimensão do nanotubo de $\mathrm{TiO} 2$, bem como, sobre a fotocorrente e a absorção de luz. Baseado nos resultados obtidos no presente trabalho pode-se concluir que a amostra que apresentou melhor desempenho fotoeletroquimico foi a $\mathrm{Nt30}$, anodizada com $30 \mathrm{~V}$ por $1 \mathrm{~h}$. Os resultados obtidos por espectroscopia de impedância eletroquímica e pela curva de corrente, indicam que a amostra Nt30 é um fotoeletrodo promissor para formar heteroestruturas baseado em nanotubos de dióxido de titânio.

\section{Agradecimentos}

A Pró-Reitoria de Pesquisa da Universidade Federal do Rio Grande do Sul PROPESQ, 2016.

Agradecimento ao apoio financeiro CAPES/UDELAR (047/2013) e FAPERGS PqG (2235-2551/14-4).

\section{REFERÊNCIAS}

1 Fernandes JA. Sensibilização de nanotubos de $\mathrm{TiO}_{2}$ com CdSe pela técnica de RF Magnetron Sputtering para aplicação em células fotoeletroquímicas. [Tese de Doutorado], Instituto de Física, Programa de Pós-Graduação em Ciência dos Materiais, Universidade Federal do Rio Grande do Sul, (RS-Brasil). 2014: 15-21, 53 e 93.

2 Fujishima A, Honda K, Electrochemical Photolysis of Water at a Semiconductor Electrode. 1972. Nature, 238, 37-38.

3 Rosa APP. Síntese e caracterização de eletrodos de nanotubos de $\mathrm{Ti} / \mathrm{TiO}_{2}$ para aplicação em fotoeletrocatálise. [Dissertação de Mestrado], Programa de PósGraduação em Química, Universidade Federal de Mato Grosso do Sul, (MS- Brasil). 2012: 24, 56-58.

4 Boery MNO, Ono E, Manfrim TP, Santos JS, Suzuki CK. Nanoestrutura de dióxido de titânio: controle do tamanho de cristalitos e teor das fases polimórficas. $19^{\circ}$ Congresso Brasileiro de Engenharia e Ciência dos Materiais. 2010: 1-2. 
5 Mahajan VK, Misra M, Raja KS, Mohapatra SK. Self-organized TiO2 nanotubular arrays for photoelectrochemical hydrogen generation: effect of crystallization and defect structures. Journal of Physics D: Applied Physics, Volume 41, Number 12. 2008: 4.

6 Paz DS. Síntese e caracterização do composto $\mathrm{TiO}_{2}$ dopado com nitrogênio e estudo de sua atividade fotocatalítica sob luz visível e irradiação solar. [Dissertação Mestrado], Programa de Pós-Graduação em Engenharia de Processos, Área de Concentração em Desenvolvimento de Processos Agroindustriais e Ambientais, Universidade Federal de Santa Maria, (RS-Brasil). 2012: 27-28.

7 Renz RP. Avaliação dos parâmetros do processo de anodização na formação de nanotubos de $\mathrm{TiO}_{2}$ e suas implicações na morfologia e na adesão ao substrato de titânio. [Tese de Doutorado], Programa de Pós-Graduação em Engenharia e Tecnologia de Materiais, Pontifícia Universidade Católica do Rio Grande do Sul, (RSBrasil). 2015: 23-24.

8 Oliveira HG. Tratamento de efluentes por energia solar: fotocatálise heterogênea eletro-assistida utilizando eletrodos de $\mathrm{TiO}_{2}$ nanocristalino e células solares. [Dissertação de Mestrado], Instituto de Química, Departamento de Físico-Química, Universidade Estadual de Campinas, (SP-Brasil) 2008: 22-24. 